\title{
THE SHILOV BOUNDARY OF THE ALGEBRA OF MEASURES ON A GROUP1
}

\author{
JOSEPH L. TAYLOR
}

If $G$ is a locally compact abelian topological group and $M(G)$ denotes the algebra of bounded regular Borel measures on $G$ under convolution multiplication, then $M(G)$ is a convolution measure algebra in the sense of [1]. In [1] we showed that the maximal ideal space of any such algebra $\mathfrak{M}$ can be represented as the semigroup $\hat{S}$ of all semicharacters on some compact abelian topological semigroup $S . S$ is called the structure semigroup of the algebra $\mathfrak{M}$. If $H=\{h \in \hat{S}:|h(s)|$ $=0$ or 1 for $s \in S\}$, then the Gelfand transform $\hat{\mu}$ of each element $\mu$ of $\mathfrak{M}$ attains its maximum modulus on $H$ (cf. [1, Theorem 3.3]). Hence the closure $\bar{H}$ of $H$ in the Gelfand topology contains the Shilov boundary of $\mathfrak{M}$. In [1] we show that when $\mathfrak{M}=M(G)$ for some nondiscrete locally compact topological group $G$, then $H$ is a proper subset of $\hat{S}$. In this paper we show that there is at least one group $G$ for which $\bar{H}$ is a proper subset of $\hat{S}$. Hence, for this group $G$, the Shilov boundary of $M(G)$ is a proper subset of the maximal ideal space of $M(G)$.

For each positive integer $n$ let $T_{n}$ be the multiplicative two point group $\{1,-1\}$ and set $G=\prod_{n=1}^{\infty} T_{n} . G$ is a compact abelian topological group. For each $n$ we let $\chi_{n}$ be the function which projects $G$ onto its $n$th coordinate. Each $\chi_{n}$ is a character in the dual group $\hat{G}$ of $G$ and each $k \in \hat{G}$ is either the identity or a finite product of distinct $\chi_{n}$ 's.

$S$ will denote the structure semigroup of $M(G)$ and $\hat{S}$ the semigroup of all continuous semicharacters on $S . \mu \rightarrow \mu_{S}$ is the natural imbedding of $M(G)$ into $M(S)$ (cf. [1, Theorem 2.3]). The Gelfand transform $\hat{\mu}$ of $\mu \in M(G)$ is described by the equation $\hat{\mu}(f)=\int f d \mu_{S}$ for $f \in \hat{S}$.

We are interested in a particular class of measures $\mu$ in $M(G)$. Let $\left\{r_{n}\right\}_{n=1}^{\infty}$ be a sequence of numbers in $[0,1)$ and for each $n$ let $\mu_{n}$ be the measure on $T_{n}$ defined by $\mu_{n}(1)=2^{-1}\left(1+r_{n}\right)$ and $\mu_{n}(-1)$ $=2^{-1}\left(1-r_{n}\right)$. Each $\mu_{n}$ is a strictly positive measure of norm one on $T_{n}$. Let $\mu$ be the measure in $M(G)$ which is the infinite product of the $\mu_{n}$. That is, if $U$ is any neighborhood in $G$ of the form $U=\left\{g \in G: \chi_{n}(g)\right.$

Received by the editors September 28, 1964.

1 This research was supported in part by the United States Army research office (Durham). 
$\left.=e_{n}, n=1,2, \cdots, m\right\}$, where $\left\{e_{n}\right\}_{n=1}^{m}$ is any $n$-tuple of 1 's and -1 's, then $\mu(U)=\prod_{n=1}^{m} 2^{-1}\left(1+e_{n} r_{n}\right)$. Note that if $k$ is any character of the form $k=\prod_{i=1}^{m} \chi_{n_{i}}$, where $\chi_{n_{i}} \neq \chi_{n_{j}}$ for $i \neq j$, then

$$
\int k d \mu=\prod_{i=1}^{m}\left[2^{-1}\left(1+r_{n_{i}}\right)-2^{-1}\left(1-r_{n_{i}}\right)\right]=\prod_{i=1}^{m} r_{n_{i}} .
$$

We denote by $\mathfrak{l}(\mu)$ the Banach space of all measures in $M(G)$ which are absolutely continuous with respect to $\mu$. The adjoint space of $\mathcal{R}(\mu)$ is $L_{\infty}(\mu)$. Hence each function $f \in \hat{S}$ determines a function $f^{\prime}$ in $L_{\infty}(\mu)$, such that $\int f d \nu_{S}=\int f^{\prime} d \nu$ for each $\nu \in \mathbb{R}(\mu)$. The map $\nu \rightarrow \nu_{S}$ is an $L$-homomorphism of $\mathfrak{R}(\mu)$ into $M(S)$ (cf. [1, Definition 1.3 and Theorem 2.3]) and its adjoint map is the map $f \rightarrow f^{\prime}$. Thus, by Theorem 1.2 of [1], $f \rightarrow f^{\prime}$ preserves pointwise multiplication and is a homomorphism of the semigroup $\hat{S}$. We are interested in characterizing the image of $\hat{S}$ in $L_{\infty}(\mu)$.

Lemma 1. Each function $f^{\prime}$ for $f \in \hat{S}$ is of the form $\lim _{m} a \prod_{n=1}^{m} \chi_{n}^{e_{n}}$, where $a$ is a constant with $|a| \leqq 1, e_{n}=0$ or 1 for $n=1,2, \cdots$, and the limit is in $L_{1}(\mu)$ norm.

Proof. If $g \in G$ we denote by $\delta_{g}$ the point measure at $g$. The function $f \in \hat{S}$ defines a multiplicative function $k$ (not necessarily continuous) on $G$ by $k(g)=\int f d\left(\delta_{g}\right)_{S}=\hat{\delta}_{g}(f)$. If $G_{0}$ denotes the subgroup of $G$ consisting of all $g$ for which $\left\{\chi_{n}(g)\right\}_{n=1}^{\infty}$ is eventually 1 , then there exists a sequence $\left\{e_{n}\right\}_{n=1}^{\infty}$ of 0 's and 1's, such that $k(g)=\prod_{n=1}^{\infty} \chi_{n}^{e_{n}}(g)$ for $g \in G_{0}$.

Now for each positive integer $m$ set $U_{m}=\left\{g \in G: \chi_{n}(g)=1\right.$ if $n \leqq m\}$ and $E_{m}=\left\{g \in G: \chi_{n}(g)=1\right.$ if $\left.n>m\right\} . E_{m}$ contains $2^{m}$ elements, $U_{m}$ is a compact neighborhood of the identity, and $\left\{g U_{m}: g \in E_{m}\right\}$ is a pairwise disjoint cover of $G$. Let $\pi_{m}$ be the characteristic function of $U_{m}$. For each $m$ we choose a collection of numbers $\left\{b_{m, g}\right\}_{g \in E_{m}}$ with $\left|b_{m, o}\right| \leqq 1$, which minimizes the number

$$
\int\left|f^{\prime}(g)-\sum_{0^{\prime} \in E_{m}} b_{m, g^{\prime}} \pi_{m}\left(g g^{\prime}\right)\right| d \mu(g) .
$$

We set $h_{m}(g)=\sum_{g^{\prime} \in E_{m}} b_{m, g^{\prime}} \pi_{m}\left(g g^{\prime}\right)$. The sequence $\left\{\int\left|h_{m}-f^{\prime}\right| d \mu\right\}_{m=1}^{\infty}$ is nonincreasing and, since the continuous simple step functions of norm $\leqq 1$ are dense in the unit ball of $L_{1}(\mu)$, it follows that this sequence converges to zero.

Fix $m$ and for $g \in E_{m}$ and $V$ a Borel set of $G$ define $\nu_{g}(V)=\mu\left(V \cap g U_{m}\right)$, then 


$$
\begin{aligned}
\nu_{\theta_{1}} & =\left\|\nu_{\sigma_{2}}\right\|^{-1}\left\|\nu_{\theta_{1}}\right\| \delta_{\sigma_{1} g_{2}}{ }^{-1} \cdot \nu_{\theta_{2}} \\
& =\prod_{x_{n}\left(\theta_{1}\right)=-1}\left(1+r_{n}\right)^{-1}\left(1-r_{n}\right) \prod_{x_{n}\left(g_{2}\right)=-1}\left(1-r_{n}\right)^{-1}\left(1+r_{n}\right) \delta_{\sigma_{1} \theta_{2}}{ }^{-1} \cdot \nu_{\sigma_{2}} .
\end{aligned}
$$

Also, it follows from the definitions of $f^{\prime}$ and $k$ that $f^{\prime}\left(g g_{1}\right)=k\left(g_{1}\right) f^{\prime}(g)$ $=\prod_{n=1}^{m} \chi_{n}{ }^{e_{n}}\left(g_{1}\right) f^{\prime}(g)$ a.e. $/ \mu$ for each $g_{1} \in E_{m}$. Choose $g_{0} \in E_{m}$ such that $\left\|\nu_{o_{0}}\right\|\left|-1 \int\right| f^{\prime}-h_{m}\left|d \nu_{g_{0}}=\min _{g \in E_{m}}\left\|\nu_{0}\right\|^{-1} \int\right| f^{\prime}-h_{m} \mid d \nu_{0}$ and let $a_{m}$ $=b_{m, 0_{0}} k\left(g_{0}\right)$ and $k_{m}=\prod_{n=1}^{m} \chi_{n}^{a^{n}}$. Then

$$
\begin{aligned}
& \int\left|f^{\prime}-a_{m} k_{m}\right| d \mu=\sum_{o_{1} \in E_{m}} \int\left|f^{\prime}-a_{m} k_{m}\right| d \nu_{o_{1}} \\
& =\sum_{\sigma_{1} \in E_{m}}\left\|\nu_{g_{0}}||-1|| \nu_{o_{1}}\right\| \int\left|f^{\prime}-a_{m} k_{m}\right| d\left(\delta_{\sigma_{10_{0}}}^{-1} \cdot \nu_{g_{0}}\right) \\
& =\sum_{0_{1} \in E_{m}}\left\|\nu_{g_{0}}\right\|-1\left\|\nu_{o_{1}}\right\| \int\left|f^{\prime}\left(g_{1} g_{0}^{-1} g\right)-a_{m} k_{m}\left(g_{1} g_{0}^{-1} g\right)\right| d \nu_{\theta_{0}}(g) \\
& =\sum_{\sigma_{1} \in E_{m}}\left\|\nu_{\theta_{0}}\right\|-1\left\|\nu_{\sigma_{1}}\right\| \int\left|k_{m}\left(g_{1} g_{0}^{-1}\right)\left(f^{\prime}(g)-a_{m} k_{m}(g)\right)\right| d \nu_{\sigma_{0}}(g) \\
& =\sum_{g_{1} \in E_{m}}\left\|\nu_{o_{0}}\right\|-1\left\|\nu_{g_{1}}\right\| \int\left|f^{\prime}-h_{m}\right| d \nu_{\sigma_{0}} \\
& \leqq \sum_{0_{1} \in E_{m}} \int\left|f^{\prime}-h_{m}\right| d \nu_{o_{1}}=\int\left|f^{\prime}-h_{m}\right| d \mu .
\end{aligned}
$$

That is, $\int\left|f^{\prime}-a_{m} k_{m}\right| d \mu \leqq \int\left|f^{\prime}-h_{m}\right| d \mu$. Hence

$$
\left\{a_{m} k_{m}\right\}_{m=1}^{\infty}=\left\{a_{m} \prod_{n=1}^{m} \chi_{n}^{e_{n}}\right\}_{m=1}^{\infty}
$$

converges in $L_{1}(\mu)$ norm to $f^{\prime}$. If $a$ is a cluster point of the sequence $\left\{a_{m}\right\}_{m}$, then $\left\{a \prod_{n=1}^{m} \chi_{n}^{e_{n}}\right\}_{m=1}^{\infty}$, also converges to $f^{\prime}$ in $L_{1}(\mu)$ norm. This completes the proof.

LEMMA 2. If $\lim \sup _{n} r_{n}<1$ and $f^{\prime}=a \lim _{m} \prod_{n=1}^{m} \chi_{n}^{o_{n}}$ as in Lemma 1, with $|a|>0$, then there exists $M$, such that $e_{n}=0$ if $n>M$. Hence $f^{\prime}=a k$ where $k=\prod_{n=1}^{M} \chi_{n}{ }^{e_{n}} \in \hat{G}$.

Proof.

$$
\int\left|\prod_{n=1}^{m-1} \chi_{n}^{e_{n}}-\prod_{n=1}^{m} \chi_{n}^{e_{n}}\right| d \mu=\int\left|1-\chi_{m}^{e_{m}}\right| d \mu=e_{m}\left(1-r_{m}\right) .
$$

Hence if $\left\{\prod_{n=1}^{M} \chi_{n}^{e_{n}}\right\}_{m=1}^{\infty}$ converges in $L_{1}(\mu)$ norm, then either $\lim \sup _{n} r_{n}=1$ or $\left\{e_{n}\right\}_{n=1}^{\infty}$ is eventually zero. 
For each positive integer $n$ let $A_{n}$ be the subset of $[0,1]$ consisting of 1 and all finite products $\prod_{i=1}^{m} r_{n_{i}}$ with $n<n_{i}$ for $i=1,2, \cdots, m$ and $n_{i} \neq n_{j}$ if $i \neq j$.

Lemma 3. If lim $\sup _{n} r_{n}<1$, then the closure of $\hat{G}$ in the weak-* topology of $L_{\infty}(\mu)$ is $\left\{a k: k \in \hat{G}\right.$ and $\left.a \in \bigcap_{n} \bar{A}_{n}\right\}$.

Proof. If $a \in \bigcap_{n} \bar{A}_{n}$ then there is a sequence $\left\{\left\{p_{i, n}\right\}_{i=1}^{m_{n}}\right\}_{n=1}^{\infty}$ of tuples of distinct integers, with $p_{i, n} \geqq n$, such that $\lim _{n} \prod_{i=1}^{m_{n}} r_{p_{i, n}}=a$. If $k \in \hat{G}$ then $k$ is a product of $\chi_{p}$ 's with $p \leqq M$ for some integer $M$; set $h_{n}=k \prod_{i=1}^{m_{n}} \chi_{p_{i}, n} \in \hat{G}$. If $U$ is any open-compact rectangle in $G$ of the form $U=\left\{g \in G: \chi_{q_{j}}(g)=\sigma_{j}\right\}$ for $j=1,2, \cdots, u$ where $\left\{\sigma_{j}\right\}_{j=1}^{u}$ is any $u$-tuple of 1 's and -1 's, then $\int_{U} h_{n} d \mu=\prod_{i=1}^{m_{n}} r_{p_{i}, n} \int_{U} k d \mu$ provided $n>q_{j}$ for $j=1,2, \cdots, u$, and $n>M$. Hence $\lim _{n} \int_{U} h_{n} d \mu$ $=\int_{U} a k d \mu$. From this fact and the fact that $\left\{h_{n}\right\}_{n=1}^{\infty}$ is uniformly bounded it follows that $\lim _{n} h_{n}=a k$ in the weak-* topology of $L_{\infty}(\mu)$.

Conversely, suppose $h$ is in the weak-* closure of $\hat{G}$ in $L_{\infty}(\mu)$. Then $h=f^{\prime}$ for some $f \in \hat{S}$ and hence, by Lemma $2, h=a k$ for some $a$ with $|a| \leqq 1$ and $k \in \hat{G}$. Let $\left\{k_{\alpha}\right\}$ be a net in $\hat{G}$ converging weak-* to $a k$. Then $\lim _{\alpha} k k_{\alpha}=a$. If $a$ is not 1 then we may assume that $k k_{\alpha}$ $=\prod_{i=1}^{m_{\alpha}} \chi_{n_{i}, \alpha}, \quad$ where $n_{i, \alpha} \neq n_{j, \alpha}$ if $i \neq j$. Then $\lim \int k k_{\alpha} d \mu$ $=\lim \prod_{i=1}^{m_{\alpha}} r_{n_{i}, \alpha}=a$. Also, since the weak-* limit of $\left\{k k_{\alpha}\right\}$ is a constant, it follows that, given $n$, eventually $n_{i, \alpha} \geqq n$ for $i=1,2, \cdots, m_{\alpha}$. Hence $a \in \bigcap_{m} \bar{A}_{m}$. This completes the proof.

THEorem 1. If $\lim \sup _{n} r_{n}<1, k \in \hat{G}$, and $0<|a|<1$, then $a k=f^{\prime}$ for some $f$ in the Shilov boundary of $M(G)$ if and only if $|a| \in \bigcap_{n} \bar{A}_{n}$.

Proof. If $|a| \in \bigcap_{n} \bar{A}_{n}$ then, by Lemma $3,|a|$ is in the weak-* closure in $L_{\infty}(\mu)$ of $\hat{G}$. It follows that there exists $h$ in the closure of $\hat{G}$ in $\hat{S}$ such that $h^{\prime}=|a|$, that is, $h$ is identically $|a|$ on the carrier of $\mu_{S}$ in $S$. Then $h$ is identically $|a|^{n}$ on the carrier of $\mu_{S}^{n}$ in $S$ for each $n$. Since $0<|a|<1$ it follows that carrier $\left(\mu_{S}^{n}\right) \cap$ carrier $\left(\mu_{S}^{m}\right)=\varnothing$ for $n \neq m$. Let $\nu(V)=\int_{V}|a|^{-1} \bar{a} \bar{k} d \mu$ for each Borel set $V$ of $G$. Then carrier $\left(\nu_{S}^{n}\right) \cap$ carrier $\left(\nu_{S}^{m}\right)=\varnothing$ for $n \neq m$, and hence

$$
\left\|\left(\nu+\delta_{e}\right) n\right\|=\left\|\sum_{m=0}^{n}\left(\begin{array}{c}
n \\
m
\end{array}\right) \nu^{m}\right\|=\sum_{m=0}^{n}\left(\begin{array}{c}
n \\
m
\end{array}\right)\left\|\nu^{m}\right\|=\sum_{m=0}^{n}\left(\begin{array}{c}
n \\
m
\end{array}\right)=2^{n},
$$

where $e$ is the identity of $G$ and $\delta_{e}$ is the point measure at $e$. Thus $\nu+\delta_{e}$ has spectral radius 2 and it follows that there exists $h_{1}$ in the Shilov boundary of $M(G)$, such that $\left|\left(\hat{\nu}+\hat{\delta}_{e}\right)\left(h_{1}\right)\right|=\left|\hat{\nu}\left(h_{1}\right)+1\right|=2$. Since $\|\nu\|=1, \hat{\nu}\left(h_{1}\right)$ must be 1 . Then $\int|a|^{-1} \bar{a} \bar{k} h_{1}^{\prime} d \mu=1$ and we conclude that $h_{1}^{\prime}=|a|^{-1} a k$ and $\left(h h_{1}\right)^{\prime}=a k$. Now the Shilov boundary is clearly invariant under multiplication by elements of $\hat{G}$ and, since the 
Shilov boundary is closed, it is invariant under multiplication by elements of the closure of $\hat{G}$ in $\hat{S}$. Hence $h h_{1}$ is in the Shilov boundary.

Conversely, suppose $a k=f^{\prime}$ where $f$ is in the Shilov boundary. By Theorem 3.3 of [1], $f$ is the limit of a net $\left\{h_{\alpha}\right\} \subset H=\{h \in \hat{S}:|h(s)|=0$ or 1 for $s \in S\}$. By Lemmas 1 and 2, there exist numbers $a_{\alpha},\left|a_{\alpha}\right|=0$ or 1 , and characters $k_{\alpha}$, such that $h_{\alpha}^{\prime}=a_{\alpha} k_{\alpha}$ for each $\alpha$. Clearly, $\lim _{\alpha} a_{\alpha}=a /|a|$ and $\lim _{\alpha} k_{\alpha}=|a| k$ in the weak-* topology of $L_{\infty}(\mu)$. Hence, by Lemma $3,|a| \in \bigcap_{n} \bar{A}_{n}$.

Theorem 2. The Shilov boundary of $M(G)$ is a proper subset of $\hat{S}$.

PROOF. If $\left\{r_{n}\right\}_{n=1}$ is chosen such that $0<\lim \sup _{n} r_{n}<1$, then there is a positive number $a \in \bigcap_{n} \bar{A}_{n}$. Then $a=f_{a}^{\prime}$ for some $f_{a} \in \hat{S}$, by Theorem 1 , where $f_{a}$ may be chosen such that $f_{a}(s) \geqq 0$ for each $s \in S$. Hence, $f_{a}^{z} \in \hat{S}$ for each complex number $z$ with $\operatorname{Re} z>0$, and $f_{a}^{z \prime}=a^{z}$. It follows that for each $b$ in the unit disc there exists $f_{b} \in \hat{S}$, such that $f_{b}^{\prime}=b$. By Theorem $1, f_{b}$ may be chosen from the Shilov boundary if and only if $|b| \in \cap \bar{A}_{n}$. However $\bigcap_{n} \bar{A}_{n} \subset\left[0, \lim \sup _{n} r_{n}\right] \cup 1$ which is a proper subset of $[0,1]$. This completes the proof.

\section{REFERENCES}

1. J. L. Taylor, The structure of convolution measure algebras, Trans. Amer. Math. Soc. 119 (1965), 150-166.

HARVARD UNIVERSITY 